\title{
Les assemblées locales au Sichuan de 1909 à 1914 : un renouvellement du politique ?
}

\author{
Monica de Togni *
}

L'analyse du processus d'élection des assemblées locales en 1909 et des relations entre ces assemblées et les magistrats de district dans leur participation active au gouvernement a pour but d'évaluer, à partir de cas concrets, la profondeur des réformes (xinzheng 新政) entreprises tardivement par la dynastie des Qing 清 (1644-1911) après la défaite des Boxeurs. Le magistrat de district était alors considéré comme représentant l'autorité du gouvernement central au niveau local, et les assemblées locales comme représentantes des intérêts locaux élues par les collectivités. Cette étude entend contribuer à une meilleure compréhension des changements en cours dans la société de l'époque ainsi que du processus de formation de l'État chinois moderne, en analysant l'interaction entre ces deux pouvoirs au cours d'une période riche en changements politiques et institutionnels. Elle vise aussi à mieux connaître les composantes sociales de l'élite locale, notamment à travers l'étude des élus - catégorie qui, comme l'a souligné Keith Schoppa ${ }^{1}$ dans les années soixante-dix, n'était pas limitée aux seuls shenshi 紳士 (notables-lettrés).

* Monica de Togni a soutenu sa thèse à l'Università Ca' Foscari de Venise le 9 février 2001 sous la direction du professeur Guido Samaraniet et y continue ses recherches au Département d'études sur l'Asie orientale. Elle tient à remercier Mme Marianne Bastid-Bruguière pour ses conseils et Delphine Spicq pour sa relecture du texte.

${ }^{1}$ Keith R. Schoppa, "The Composition and Functions of the Local Elite in Szechwan, 1851-1874". 
Les rapports entre assemblées et magistrats ont été analysés ici à partir de documents d'archives locales et de revues de l'époque, notamment le Sichuan guanbao 四川官報, en limitant l'horizon géographique à une région spécifique : le Sichuan. La période choisie s'étend des dernières années des Qing à 1914, année importante qui voit l'introduction de nouvelles réglementations pour les concours des magistrats comme pour l'élection des assemblées locales. Il s'agit aussi d'une période pendant laquelle s'amorce la mise en place effective d'une autonomie locale, conçue dans le but de confier aux représentants élus par la collectivité des responsabilités réelles dans l'administration.

Jusqu'à présent, l'intérêt porté aux assemblées locales de la fin des Qing se bornait, en Occident, à la recherche des signes reconnaissables d'un processus de démocratisation alors à l'œuvre en Chine ${ }^{2}$, ainsi qu'à l'analyse du point de vue des fonctionnaires de la capitale et du gouvernement central ${ }^{3}$, tandis que l'attention des savants chinois se portait sur l'étude des assemblées. Cette analyse consistait principalement à examiner la constitution et les composantes sociales de ces assemblées, en utilisant comme sources principales les monographies locales (difang $z h i$ 地方志) ${ }^{4}$.

L'ensemble des réformes introduites à la fin des Qing aboutit à la transformation de l'empire en monarchie constitutionnelle. Cette transformation est sanctionnée en premier lieu par un édit du $1^{\text {er }}$ septembre $1906^{5}$,

${ }^{2}$ John Fincher, Chinese Democracy: The Self-Government Movement in Local Provincial and National Politics, 1905-1914.

${ }^{3}$ Roger Thompson, China's Local Councils in the Age of Constitutional Reform, 1898-1911.

${ }^{4}$ Cf. Zhang Yufa 張玉法, «Qingmo Minchu de Shandong difang zizhi »清末民初的 山東地方自治, ou voir, pour ce qui concerne le Sichuan, Liu Zhuanying 劉傳英, 《Difang zhengzhi de yanbian》地方自治的演變, et Wang Di 王笛, Kuachu fengbi de shijie: Changjiang shangyou quyu shehui yanjiu, 1644-1911 跨出封閉的世界: 長江上游區域社會研究.

${ }^{5}$ Gugong bowuyuan Ming Qing dang'an bu (éd.) 故宮博物院明清檔案部, Qingmo choubei lixian dang'an shiliao 清末䇾備立麦檔案史料, p. 43-44. 
promulgué après le retour en Chine des fonctionnaires envoyés au Japon, en Europe et aux États-Unis afin d'examiner les différents systèmes de gouvernement ; un second édit daté du 27 août 1908 définit, entre autres, les Principes constitutionnels et le Programme préparatoire en neuf ans ${ }^{6}$. Parmi les mesures proposées, ce programme prévoit la mise en place d'organes de l'autonomie locale (difang zizhi 地方自治) à deux échelons : l'échelon inférieur constitué par les assemblées délibérantes des villes, bourgs et villages (cheng zhen xiang yishihui 城、鎮、鄉議事會) et par des conseils administratifs (cheng zhen dongshihui xiangdong 城、鎮董事會、鄉董), et l'échelon supérieur constitué d'assemblées (yishihui 議事會) et de conseils (canshihui 參事會) dans les préfectures, les sous-préfectures, les départements et les districts (fu ting zhou xian 府、廳、州、影系 $)^{7}$. Ce système institutionnel a été emprunté au Japon par Yuan Shikai 袁世凱 (1859-1916), qui l'avait expérimenté à Tianjin 天津 en 1906-1907 sur une sollicitation de la Cour, lorsqu'il était gouverneur du Zhili 直隸. Une telle bipartition vise à la séparation, au moins théorique, des fonctions législative et exécutive entre les assemblées délibérantes, qui se réunissent seulement à certaines périodes de l'année, et les conseils administratifs, qui sont permanents ${ }^{8}$.

Cette expérimentation fut ensuite utilisée par la Cour qui imaginait une application d'une manière uniforme à tout l'empire. Elle entraîna la constitution des premières assemblées locales, sur la base de deux règlements : le Règlement pour l'autonomie locale des villes, bourgs et villages,

\footnotetext{
${ }^{6}$ Ibid., p. 61-68.

${ }^{7}$ Le niveau de la préfecture n'était pas prévu par le Programme préparatoire, mais il est inclus dans le règlement promulgué en 1910 ; cf. Sichuan guanbao 四川官報, 1910,6, zouyi 奏議 (rapports à l'empereur).

${ }^{8}$ On utiliscra dans cet article l'expression «assemblées locales » pour se référer à l'ensemble formé par ces deux institutions. Pour la dette contractée avec le Japon et une analyse du processus d'élaboration du système chinois, voir Roger Thompson, $o p$. cit., p. $37-52,86$.
} 


\section{Notes de recherche}

promulgué le 18 janvier 1909 (Guangxu 光緒 34/12/27) ${ }^{9}$, et le Règlement pour l'autonomie locale des préfectures, sous-préfectures, départements et districts promulgué l'année suivante, le 6 février 1910 (Xuantong 宣統 $1 / 12 / 27)^{10}$, conformément au Programme préparatoire.

Le poids de ces mesures de renouvellement politique est important si l'on considère que ces règlements constituèrent la première reconnaissance statutaire de la participation de la collectivité à son propre gouvernement par le biais de représentants élus. Apparut en outre une forme de pénétration de l'État à un niveau inférieur au district, échelon auquel s'arrêtait alors la présence formelle de la puissance publique. D'une certaine façon, à travers la concession officielle d'une plus grande autonomie locale, le gouvernement central chercha à étendre son contrôle à un échelon qui auparavant lui échappait. Cette extension de l'influence du politique dans le domaine social, jusqu'alors non concerné par l'intervention étatique, permet d'analyser la façon dont le dialogue entre État et société s'est modifié.

La constitution des assemblées étant un phénomène tout à fait nouveau, il a fallu préparer ceux qui étaient impliqués dans ce processus et diffuser les idées nouvelles qui avaient engendré ces changements. La solution adoptée par Yuan Shikai fut étendue au reste du pays au moyen d'un règlement. Des centres d'étude de l'autonomie locale (zizhi yanjiusuo 自治研究所) furent créés. Le premier, composé d'étudiants chinois rentrés du Japon après des études de droit, vit le jour à Tianjin en octobre $1906^{11}$. Le règlement qui avalisait ces centres - le Règlement pour les centres d'étude sur l'autonomie locale (Zizhi yanjiusuo zhangcheng 章程) - fut promulgué par le gouvernement central le $1^{\text {cr }}$ mai 1909 (Xuantong $1 / 03 / 16)^{12}$. L'objectif de ces centres est expliqué dans la préface du Règlement : il s'agissait de préparer des personnes à envoyer dans leur district ou

\footnotetext{
${ }^{9}$ Gugong bowuyuan Ming Qing dang'an bu (éd.), p. 724-741.

${ }^{10}$ Sichuan guanbao, 1910, 6, zouyi, p. 1a-9a.

${ }^{11}$ Cf. Roger Thompson, op. cit., p. 47.

${ }^{12}$ Cf. Gugong bowuyuan Ming Qing dang'an bu (éd.), op. cit., p. 745-748.
} 
préfecture de naissance afin de diffuser les connaissances relatives aux réformes et à l'autonomie locale ; on considérait le peuple encore incapable de participer d'une manière autonome au nouveau système de gouvernement, et il fallait donc l'instruire et le responsabiliser. Les associations, écoles et centres d'études créés avant la promulgation de ce Règlement durent s'y conformer. En fait, la révision du Code (Da Qing lüli 大清律例) ${ }^{13}$, déjà entamée par le gouvernement des Qing, faisait partie du processus de transformation vers un État moderne, doté de règlements et de lois organiques uniformes. Il fallait de ce fait éviter toute réglementation dérogatoire.

Cette nouvelle réglementation peut être interprétée d'au moins deux façons différentes. On peut la considérer d'abord comme la reconnaissance de la nécessité d'un soutien aux « sujets » dans le processus de leur transformation en citoyens : il s'agit de les convaincre de participer activement au gouvernement de la collectivité. On peut y voir d'autre part le moyen de restreindre les nombreuses initiatives de la partie de la population la plus active dans le champ du politique. Le procédé d'enregistrement des électeurs et des éligibles témoigne en effet largement de cette nécessité de convaincre les citoyens de participer à la vie politique. Ainsi, un magistrat du district de Chengdu invita dans le Sichuan guanbao ceux qui en avaient le droit à s'inscrire sur les registres électoraux ${ }^{14}$ et leur expliqua qu'ils

${ }^{13}$ À propos de la réforme des règlements et des lois, cf. Ichiko Chuzo, "Political and Institutional Reform, 1901-1911", p. 408-410, et Zhang Jinfan 張㿟藩, Qing chao fazhi shi 清朝法製史, p. 709-716.

${ }^{14}$ Selon les principales caractéristiques expliquées dans l'art. 16 du Règlement pour l'autonomie locale de villes, bourgs et villages, le droit de vote est accordé à tous les hommes de nationalité chinoise (benguo guoji 本國國籍), de plus de vingt cinq ans, résidant depuis au moins trois ans dans l'endroit où ils votent, ou résidant au dehors de leur lieu de naissance mais payant des impôts annuels d'au moins deux yuan 元. Des dérogations sont prévues pour ceux dont les qualités morales sont reconnues par la communauté locale, même s'ils sont résidents depuis moins de trois ans ou s'ils payent des impôts inférieurs à deux yuan, ainsi que pour les contribuables qui versent les 


\section{Notes de recherche}

n'avaient pas à craindre une surcharge d'impôts. Au contraire, il insista sur le droit de chaque citoyen de participer aux organes de l'autonomie locale, et donc de sauvegarder aussi ses intérêts, et sur le devoir de payer ses impôts, qui est associé à ce droit ${ }^{15}$. De tels appels laissent penser que cette nouvelle forme de participation était encore étrangère à la société chinoise et que les centres d'étude de l'autonomie locale - organisés par les notables locaux en association avec les fonctionnaires et précédant les partis, qui venaient tout juste de démarrer leurs activités sans recueillir encore beaucoup de militants - étaient nécessaires pour aider à diffuser une conscience citoyenne parmi la population. D'un autre côté, la promulgation d'un règlement se révélait également nécessaire afin de mettre de l'ordre dans la pléthore d'organisations qui s'étaient créées à l'initiative de citoyens au niveau local et dont le gouvernement central à Pékin avait du mal à suivre les initiatives ${ }^{16}$. À l'heure actuelle, on ne dispose pas encore d'une vision détaillée de toutes les initiatives entreprises au Sichuan; on peut néanmoins affirmer que cette province connaissait alors un important activisme local, comme le montrent les quelques cas suivants.

Dans les numéros du Sichuan guanbao de 1908 apparaissent plusieurs exemples de la participation de citoyens à l'élaboration d'une nouvelle relation entre eux-mêmes et les institutions gouvernementales ; ainsi, celui d'une association pour l'autonomie locale (zizhihui 自治會) ${ }^{17}$ créée par des étudiants d'un collège de Shunqing 順慶 ${ }^{18}$, pour qui l'autonomie d'une collectivité ne peut exister sans celle de chaque individu. Les étudiants qui la composent doivent donc participer activement, en diffusant

contributions les plus importantes, sans restriction d'âge ou de lieu de résidence. $\mathrm{Cf}$. Gugong bowuyuan Ming Qing dang'an bu (éd.), op. cit., p. 730.

${ }^{15}$ Cf. Sichuan guanbao, 1909, 28, yanshuo 演說 (discours), p. 1a-b.

${ }^{16}$ Cf. Roger Thompson, op. cit., p. 58-66.

${ }^{17} \mathrm{Ce}$ mot est ensuite également utilisé pour désigner les assemblées locales, donnant parfois lieu à des problèmes de compréhension. Voir par exemple Sichuan sheng dang'an guan (éd.) 四川省檔案館, Baxian xianshu 巴縣縣署, 193.1011, p. 3-4.

${ }^{18}$ Sichuan guanbao, 1908, 27, xinwen 新聞 (nouvelles), p. $4 a$. 
dans leurs villages, lorsqu'ils rentrent chez eux pendant les vacances, leurs nouvelles connaissances sur l'autonomie locale. L'initiative vient aussi des magistrats de district, comme celui de $\mathrm{Pi}$ 猝, qui sollicite les notables locaux pour créer un centre d'étude de l'autonomie locale au printemps 1908. Les notables qui suivent les cours dans ce centre payent des frais s'élevant à trois yuan ${ }^{19}$. Il est assez remarquable de trouver des notables disposés à suivre des cours et qui, en plus, acceptent de payer une somme assez considérable pour l'époque.

L'activisme des communautés locales semble avoir parfois inquiété le gouvernement central au point que, en plusieurs occasions, les autorités ont rappelé que les organes de l'autonomie locale n'avaient que des fonctions auxiliaires par rapport à l'administration étatique, à laquelle ils restaient subordonnés. Le gouvernement central n'en était pas moins soucieux d'expurger chez les fonctionnaires le préjugé selon lequel l'autonomie locale constituait une ingérence dans leurs compétences, bien qu'il on considérait les élus comme des «novices sans expérience» de la vie politique et qu'il confiait plutôt aux fonctionnaires un large pouvoir de contrôle ${ }^{20}$.

La fonction de magistrat de district subit un changement important avec l'adoption d'un système de monarchie constitutionnelle, qui posait le principe de la séparation des pouvoirs. Le magistrat se trouva alors, au moins théoriquement, dépouillé de son pouvoir judiciaire, confié à des tribunaux nouvellement créés ${ }^{21}$. C'est dans cette perspective de

\footnotetext{
${ }^{19}$ Sichuan guanbao, 1909, 24, xinwen, p. 2b-3a.

${ }^{20}$ Sichuan guanbao, 1910,3, gongdu 公牌 (documents officiels), p. 2a-b.

${ }^{21}$ Le pouvoir judiciaire du magistrat de district est transféré aux tribunaux dont la structure est la suivante :

- tribunaux locaux (chuji shenpan ting 初級審判廳) et procures locales (chuji jiancha ting 初級檢察廳) dans les villages ;

- cours de district (difang shenpan ting 地方害判廳) et procures de district (difang jiancha ting 地方檢察廳) dans les villes de district;
} 
renouvellement institutionnel qu'il faut interpréter la décision d'adjoindre au magistrat des organes de l'autonomie pour la gestion des affaires locales. Cette contrainte ne représente pas seulement une mesure politique, mais vise à améliorer l'efficacité d'un système administratif essoufflé par les trop nombreuses tâches données à un personnel trop réduit ${ }^{22}$.

En fait, le magistrat de district bénéficiait déjà de l'aide de l'élite locale dans l'exercice de ses fonctions. Représentant de l'autorité impériale, le magistrat devait préserver l'harmonie et éviter l'accumulation de procès en attente de jugement ou le défaut dans la collecte des taxes. De la même façon, il était responsable de ses employés et devait contrôler leurs activités $^{23}$, tâche difficile, comme en témoignent de nombreux manuels de fonctionnaires édités au cours de la dynastie. On trouve au Sichuan, avant la mise en place de l'autonomie locale, un exemple intéressant de coopération entre magistrats et élite locale contre les «employés-vermines " : il s'agit du Bureau des Trois frais (Sanfei ju 三費局) qui avait été constitué

- cours supérieures (gaodeng shenpan ting 高等審判廠) et procures supérieures (gaodeng jiancha ting 高等檢察廳) dans la capitale et dans les chefs-lieux des provinces.

Ces organes référaient à la Cour suprême de justice (Dali yuan 大理院) et à la Procure générale (Zong jiancha ting 總檢察廳) de la capitale.

Selon le Programme préparatoire du 27 août 1908, la création de ces organes judiciaires est prévue pour les années 1909-1910 dans les chefs-lieux de province et dans les Ports ouverts, pour 1911-1913 dans les chefs-lieux de préfecture, sous-préfecture, département et district, pour 1913-1915 dans les bourgs et les villages. Cf. Ichiko Chuzo, op. cit, p. 395 ; H. S. Brunnert, V. V. Hagelstrom, Present Day Political Organization of China, n. 215, 222, 758, 759, 760-765 ; cf. aussi Gugong bowuyuan Ming Qing dang'an bu (éd.), op. cit., p. 62-66.

${ }^{22}$ Bien que la population ait augmenté considérablement sous les Qing, la fonction publique continue de compter un nombre, presque inchangé, d'environ vingt mille fonctionnaires seulement. Cf. J. K. K. Fairbank, "Introduction: The Old Order", p. 12. ${ }^{23}$ Voir les deux études classiques sur la fonction du magistrat de district et sur le gouvernement local : John R. Watt, The District Magistrate in Late Imperial China ; Ch'u T'ung-tsu, Local Government in China under the Ch'ing. Cf aussi l'article de Pierre-Étienne Will dans ce volume d'Études chinoises. 
pendant la deuxième moitié du XIX ${ }^{\mathrm{e}}$ siècle à la requête des notables locaux pour échapper aux abus des employés chargés de l'arrestation des coupables et de leur transfert vers les tribunaux ${ }^{24}$.

Avec les réformes, le soutien informel de l'élite aux magistrats reçoit une délimitation précise. Définie dans l'article 5 du Règlement sur l'autonomie locale de villes, bourgs et villages de 1909, le champ d'intervention des assemblées se révèle assez vaste :

- instruction des enfants

- santé publique : associations pour la lutte contre l'opium, etc.

- travaux publics: construction et entretien des routes, construction de ponts, entretien des canaux, construction d'édifices publics, éclairage public, etc.

- soutien aux activités agricoles, productives et commerciales

- activités philanthropiques : soutien aux démunis, assistance aux veuves, instruction des orphelins, quête de fonds pour les greniers publics (yicang 義倉), organisation d'associations et activités de secours en cas de famine, sauvegarde des monuments ou ruines anciennes, etc.

- services publics: transports (tramway), éclairage des rues, adduction d'eau, etc.

- collecte et gestion des fonds nécessaires à la mise en place de l'autonomie

- ce qui traditionnellement était dévolu à la gestion des notables ${ }^{25}$.

Ce dernier point souligne les contradictions du projet réformiste des dernières années des Qing avec, d'un côté, la nécessité d'uniformiser les lois et les normes, et de l'autre celle de respecter les spécificités locales, particulièrement fortes dans les provinces chinoises au début du $\mathrm{XX}^{\mathrm{e}}$ siècle, lorsque les moyens de communications étaient réduits et en particulier

24 Les «trois frais" concernent les cas judiciaires et ils sont liés aux enquêtes, à l'arrestation des coupables et à leur escorte aux procès. Voir Zhou Xun 周詢, Shu hai congtan 蜀海叢談, juan 2, p. 44a-45b, et Bradly W. Reed, "Gentry Activism in Nineteenth-Century Sichuan: The Three-fees Bureau",

${ }^{25}$ Gugong bowuyuan Ming Qing dang'an bu (éd.), op. cit., p. 728-729. 
au Sichuan où la principale et presque unique voie de communication était le Fleuve bleu.

Une question importante est celle de la composition sociale de l'électorat et des personnes éligibles. Une étude sur cette question permettrait de mieux comprendre les changements en cours à l'époque dans les relations entre la sphère du politique et la société. Si l'information est assez riche sur les élus des assemblées provinciales ${ }^{26}$, les données disponibles sur les assemblées de l'autonomie locale sont, elles, parcellaires et incomplètes.

Dans son ouvrage de 1995, Roger Thompson a analysé un groupe de 327 électeurs du district de Huichang 會昌 au Jiangxi 江西 dont 129, soit environ $40 \%$, sont inclus au titre de leur patrimoine, étant dépourvus d'un diplôme officiel (ils ne font donc pas partie des shenshi) ${ }^{27}$. On retrouve la même proportion au Sichuan pour un groupe de 49 élus aux conseils des bourgs du district de $\mathrm{Ba} 巴^{28}$. Ces exemples montrent que les personnes auxquelles étaient confiées des tâches de responsabilité dans le gouvernement local étaient choisies sur des critères d'instruction et de patrimoine, le suffrage étant censitaire, ainsi qu'en fonction de leur participation et de leur expérience dans des activités de soutien à l'administration

\footnotetext{
${ }^{26}$ Les recherches sur ce sujet sont nombreuses. $\grave{A}$ titre d'exemple, pour un tableau général, voir Chang P'eng-yuan, "Provincial Assemblies: The Emerging of Political Participation, 1909-1914" ; Min Tu-gi, National Polity and Local Power. The Transformation of Late Imperial China; pour un tableau de l'assemblée provinciale du Sichuan, voir Liu Zhuanying 劉傳英, op. cit., p. 335-392 ; Tang Zongyao 唐宗堯, 《Lixianpai ren he Sichuan ziyiju de chengli 》立憲派人和四川諮議局的成立； Zhang Huichang 張惠昌, 《Lixianpai ren he Sichuan ziyiju»立憲派人和四川 諧議局.

${ }^{27}$ Roger Thompson, op. cit., p. 68, 140.

${ }^{28}$ Sichuan sheng dang'an guan (éd.), Ba xian xianshu, 193.20.
} 
locale, ce qui était le cas des chefs de village (lizheng 里正), des chefs des milices locales (lianzheng 練正), etc. ${ }^{29}$.

L'examen des rôles respectifs du magistrat de district et des assemblées locales dans le processus décisionnel laisse apparaître une attitude des uns envers les autres généralement plus tournée vers la collaboration que vers la dissension. Une des raisons en est que ni la population ni les magistrats ne semblent satisfaits des changements introduits par les réformes. En effet, suite à la réduction du nombre de leurs fonctions, les magistrats ont moins d'occasions de prélever des fonds pour la gestion de leurs yamen 衙門, comme dans le cas de la réforme du système judiciaire et de la révision ou réduction des peines auxquelles on imputait souvent, à l'époque, l'augmentation exponentielle du banditisme ${ }^{30}$. On note, à propos de ce problème du banditisme, une collaboration active entre les magistrats et les assemblées pour aboutir à une solution. Les délégués des assemblées étaient conscients que leur intervention dans le domaine de la défense locale n'était pas inscrite dans le règlement, mais ils la justifiaient par les sollicitations dont ils faisaient l'objet de la part de la population ${ }^{31}$. Je n'ai pas trouvé de témoignages de magistrats s'opposant aux mesures proposées, sauf dans un cas d'achat d'armes lors du Mouvement pour la protection du chemin de fer, en 1911, durant lequel le gouvernement central avait interdit l'achat de matériel de défense par du personnel non habilité ${ }^{32}$. Tout change pendant les premières années de la République, lorsque le banditisme prend des proportions telles que toute interrogation sur la légitimité de l'intervention des assemblées en matière de défense locale est momentanément écartée.

\footnotetext{
${ }^{29}$ Voir Monica de Togni, Il governo locale in Cina (1909-1914). Funzionari, elezioni e assemblee nel Sichuan, p. 116-118.

${ }^{30}$ Sichuan sheng dang'an guan (éd.), Ba xian dang'an, Qing 清 6, Xuantong, 4.514, p. 1-2.

${ }^{31}$ Ibid., 2.187 .

${ }^{32}$ Ibid., 3.391, p. 1-3.
} 


\section{Notes de recherche}

Les modalités d'intervention des notables locaux - au sens large de ceux qui furent politiquement actifs au niveau local - sont le fruit d'une collaboration avec les représentants de l'État en place avant les réformes. La formalisation statutaire des notables au travers des organes de l'autonomie locale leur donne une conscience plus profonde de leur rôle de représentants d'une communauté.

Les premiers résultats de cette recherche, bien que limités, mettent en évidence des changements qu'il faudrait analyser pour mieux comprendre la naissance des mouvements politiques du début de la République. Un autre élément important à considérer pour mieux comprendre les dynamiques de la socialisation politique est l'influence exercée par les associations (sociétés secrètes, lignages) qui structuraient la société traditionnelle. La Société des aînés et des anciens (Gelaohui 哥老會) ${ }^{33}$ était particulièrement présente au Sichuan et, considérant son rôle dans la révolution de 1911, il est difficile de supposer qu'elle ait eu un rôle secondaire au niveau local. Les lignages avaient une fonction politique reconnue surtout dans les provinces côtières, telles que le Guangdong 廣東 et le Fujian 福建, mais le Sichuan ne nous paraît pas complètement étranger à ce phénomène. Ces différents thèmes mériteraient des études plus poussées, notamment dans un environnement particulier comme le Sichuan, ce qui fera l'objet de notre prochaine étude.

Le tableau de la situation prévalant au Sichuan au début de la République qui est dressé dans cet article est fondé sur des documents d'archives et des revues. Des analyses d'archives et de revues d'autres

\footnotetext{
${ }^{33}$ Mieux connue au Sichuan sous la dénomination de paoge 袍哥. Nous nous bornons ici à indiquer quelques-unes des études : He Yimin 何一民, «Huidang yu xinhai geming 》會黨與辛亥革命; Hou Yun 後雲, 《Xinhai geming shi de Sichuan Gelaohui 》辛夜革命史的四川歌老會; Zhang Jie 張傑, 《Minguo Chuansheng tufei, paoge yu junfa de lianxi 》民國川省士匪, 袍哥與軍閣的眑係; Zhao Qing 趙清, 《Zhongshi dui paoge, tufei he junfa shi de yanjiu » 重視對袍歌, 土匪和軍閥史的研究.
} 
provinces de Chine nous diront si cette nouvelle forme de dialogue et d'interpénétration entre État et société était aussi répandue dans le reste du pays.

\section{Bibliographie}

Brunnert H. S., Hagelstrom V. V., Present Day Political Organization of China, Taibei : Chengwen, 1978 (éd. originale Shanghai, 1912).

Chang P'eng-yuan, "Provincial Assemblies: The Emerging of Political Participation, 1909-1914", Chinese Studies in History, 17, 3 (1984), p. 3-28.

Ch'ü T'ung-tsu, Local Government in China under the Ch'ing, Stanford : Stanford University Press, 1969.

de Togni Monica, Il governo locale in Cina (1909-1914). Funzionari, elezioni e assemblee nel Sichuan, thèse de doctorat (Venezia 2001).

Fairbank J. K. K., "Introduction: The Old Order", in J.J.K. Fairbank (éd.), The Cambridge History of China, Cambridge, London, New York: Cambridge University Press, 1978, vol. 10, p. 1-34.

Fincher John, Chinese Democracy: The Self-Government Movement in Local Provincial and National Politics, 1905-1914, London, Croom Helm, New York : St. Martin's Press, 1981.

Gugong bowuyuan Ming Qing dang'an bu (éd.) 故宮博物院明清檔案部, Qingmo choubei lixian dang'an shiliao 清末籌備立憲檔案史料 (Documents d'archives sur les préparatifs constitutionnels à la fin des Qing), Beijing : Zhonghua shuju, 1979.

He Yimin 何一民, “Huidang yu xinhai geming》會黨與辛亥革命 (Les sociétés secrètes et la révolution de 1911), in Wei Yingtao 魏瀛濤 (éd.), 
Xinhai geming yu Sichuan shehui 辛亥革命與綸川社會 (La révolution de 1911 et la société du Sichuan), Chengdu : 1991, p. 171-210.

Hou Yun 後雲, «Xinhai geming shi de Sichuan Gelaohui 》辛亥革命 史的四川歌老會 (La Société des ainés et des anciens au Sichuan pendant la Révolution de 1911), Sichuan shiyuan xuebao 四川師院學報, 1983, 1.

Ichiko Chuzo, "Political and Institutional Reform, 1901-1911", in J. K. K. Fairbank (éd.), The Cambridge History of China, Cambridge, London, New York : Cambridge University Press, 1980, vol. 11, p. 375-415.

Liu Zhuanying 劉傳英, 《Difang zhengzhi de yanbian》地方自治的演變 (L'évolution du gouvernement local), in Wei Yingtao (éd.) 魏紊濤, Xinhai geming yu Sichuan shehui 辛亥革命與四川社會 (La révolution de 1911 et la société du Sichuan), Chengdu : 1991, p. 335-392.

Min Tu-gi, National Polity and Local Power. The Transformation of Late Imperial China, Cambridge (Mass.) : Harvard University Press, 1989.

Reed Bradly W., "Gentry Activism in Nineteenth-Century Sichuan: The Three-fees Bureau", Late Imperial China, 20, 2 (1999), p. 99-127.

Schoppa Keith R., "The Composition and Functions of the Local Elite in Szechwan, 1851-1874", Ch'ing-shi wen-t'i, 2, 10 (1973), p. 7-23.

Sichuan guanbao 四川官報 (Journal des fonctionnaires du Sichuan), 1908, ce 筮 $27 ; 1909$ ce 24, $28 ; 1910, c e 3,6$.

Sichuan sheng dang'an guan 四川省檔案館 (Archives provinciales du Sichuan), Ba xian xiansh $u$ 巴縣縣署 (Administration du district de Ba) $193.20 ; 193.1011$.

Sichuan sheng dang'an guan 四川省檔案館 (Archives provinciales du Sichuan), Ba xian dang'an 巴縣檔案 (Archives du district de Ba), Qing 清 6 , Xuantong 宣統 $2.187 ; 3.391 ; 4.514$.

Tang Zongyao 唐宗堯, «Lixianpai ren he Sichuan ziyiju de chengli » 立憲派人和四川諮議局的成立 (Les constitutionnels et l'établissement de l'assemblée provinciale du Sichuan), in Sichuan sheng zhengxie wenshi ziliao weiyuanhui (éd.) 四川省政協文史資料委員會, Sichuan wenshi ziliao jicui 四川文史資料集粹 (Recueil de documents histori- 
ques sur le Sichuan), Chengdu : Sichuan renmin chubanshe, 1996, vol. 1, p. 114-132.

Thompson Roger, China's Local Councils in the Age of Constitutional Reform, 1898-1911, Cambridge (Mass.) : Harvard University Press, Council on East Asian Studies, 1995.

Wang Di 王笛, Kuachu fengbi de shijie: Changjiang shangyou quyu shehui yanjiu，1644-1911 跨出封閉的世界：長江上游區域社會研究 (L'ouverture d'un monde clos : étude sociale sur la région du hautYangzi, 1644-1911), Beijing : Zhonghua shuju, 1993.

Watt John R., The District Magistrate in Late Imperial China, New York, London : Columbia University Press, 1972.

Zhang Huichang 張惠昌, «Lixianpai ren he Sichuan ziyiju»立憲派人 和四川諮議局 (Les constitutionnels et l'assemblée provinciale du Sichuan), in Zhongguo renmin zhengzhi xieshang huiyi quanguo weiyuanhui \& Wenshi ziliao yanjiu weiyuanhui (éd.) 中國人民 政治協商會議全國委員會, 文史資料研究委員會, Xinhai geming huiyi $l u$ 辛㑞革命回憶錄 (Mémoires sur la révolution de 1911), Beijing: Zhonghua shuju, 1962, vol. 3, p. 145-173.

Zhang Jie 張桀, «Minguo Chuansheng tufei, paoge yu junfa de lianxi » 民國川省土匪、袍哥與軍閥的聯係 (Les relations entre les Seigneurs de la guerre, la Société des aînés et des anciens et les bandits dans la province du Sichuan pendant la République), Jiangsu shehui kexue 江蘇社會科學, 1991, 3 .

Zhang Jinfan 張晉藩, Qingchao fazhi shi 清朝法製史 (Histoire du système juridique de la dynastie Qing), Beijing : Falü chubanshe, 1994.

Zhang Yufa 張玉法, "Qing mo Min chu de Shandong difang zizhi » 清末民初的山東地方自治 (L'autonomie locale au Shandong à la fin des Qing et au début de la République), Zhongyang yanjiuyuan jindaishi yanjiusuo jikan 中央研究院近代史研究所集刊, 6 (1978), p. 159-184.

Zhao Qing 趙清, “Zhongshi dui paoge, tufei he junfa shi de yanjiu» 重視對袍哥、士匪和軍閥紕的研究 (Considérations à propos de la recherche historique sur la Société des aînés et des anciens, les bandits 


\section{Notes de recherche}

et les Seigneurs de la guerre), Sichuan daxue xuebao 四川大學學報, $1990,2$.

Zhou Xun 周詢, Shu hai congtan 䖲海叢談 (Mélanges sur le Sichuan), Taibei : Wenhai chubanshe, 1966 (éd. originale s.l., 1948). 